\title{
Modelisation de la Dynamique du Trait de Cote sur une Portion de la Cote Ouest Cameroun Allant de Batoke a Seme Beach par Imagerie Landsat de 1979 a 2018
}

Kouekam Kengap Arnaud, M.sc

Station Spécialisée de

Recherche Halieutique et Océanographique de Limbé, Cameroun

Fowe Paul Gustave, M.sc

Togue Kamga Fulbert, PhD

Institut des Sciences Halieutiques Douala, Cameroun

Ngueguim Jules Romain, PhD

Station Spécialisée de

Recherche Halieutique et Océanographique de Limbé, Cameroun

Tsague Junior Stephan, M.sc

Institut des Sciences Halieutiques Douala, Cameroun

Doi:10.19044/esj.2019.v15n24p165 URL:http://dx.doi.org/10.19044/esj.2019.v15n24p165

\section{Resume}

La présente étude a pour objectif général de contribuer à la connaissance actuelle et future de la dynamique du trait de côte d'une portion de la côte Ouest-camerounaise à l'aide des images satellitaires Landsat 3, 7 et 8 de 1979 à 2018. Pour atteindre cet objectif, les images ont subi un prétraitement à travers des corrections atmosphériques à l'aide du logiciel ENVI 5.1; ce qui a permis la digitalisation des traits de côtes de la zone d'étude contenus dans les différentes images à l'aide du logiciel ArcGIS version 10.1 puis le calcul statistique des vitesses d'évolution du trait de côte à différentes date s'est effectué à l'aide de DSAS version 4.3. Les principaux résultats obtenus nous montrent deux tendances évolutives du linéaire côtier d'intérêt : sur le long terme, l'étude a donné un recul du trait de côte d'un taux moyen annuel égal à $-1,09 \mathrm{~m} / \mathrm{an}$ entre 1979 et 2018 et à moyen terme, l'étude montre une avancée du trait de côte avec une vitesse moyenne d'évolution égale à $+0,75 \mathrm{~m} / \mathrm{an}$ entre 2000 et 2018 . Dans cette même période, la zone a connu à court terme une situation constante d'érosion entre 2000-2005 et 2005-2008 avec un taux moyen de $-1,12 \mathrm{~m} / \mathrm{an}$, une légère stabilité entre 2008 et 2012 avec une vitesse de $-0,08 \mathrm{~m} / \mathrm{an}$, tandis que de 2012 à 2016, elle a subi un engraissement en moyenne de $+2,44 \mathrm{~m} / \mathrm{an}$. Une prévision du phénomène en 
2028 a donné une position du trait de côte de 10,90 m par rapport à sa position actuelle, ce qui pourrait mettre en péril des infrastructures hôtelières telles que : Seme Beach Hôtel ; Madison Beach ; Etisah Beach et les campements des pêcheurs du village Batoké pendant les épisodes de marées de vives eaux.

Mots clés: Engraissement, Erosion, Stabilité, Télédétection, Vitesse d'évolution

\title{
Modeling the Dynamics of the Coastline of a Portion on the Western Coast Cameroon from Batoke to Seme Beach by Imaging Landsat from 1979 to 2018
}

\author{
Kouekam Kengap Arnaud, M.sc \\ Station Spécialisée de \\ Recherche Halieutique et Océanographique de Limbé, Cameroun \\ Fowe Paul Gustave, M.sc \\ Togue Kamga Fulbert, PhD \\ Institut des Sciences Halieutiques Douala, Cameroun \\ Ngueguim Jules Romain, PhD \\ Station Spécialisée de \\ Recherche Halieutique et Océanographique de Limbé, Cameroun \\ Tsague Junior Stephan, M.sc \\ Institut des Sciences Halieutiques Douala, Cameroun
}

\begin{abstract}
The general objective of this study is to contribute to the actual and future acknowledging of the coastline dynamics of a portion of the WestCameroon coast using Landsat satellite images 3, 7 and 8 from 1979 to 2018. To achieve this objective, the images were pre-treated through atmospheric corrections using the ENVI 5.1 software, which enabled the digitalization of the coastlines of the study zone in the different images using the ArcGIS version 10.1 software and then the statistical computation of the speeds of evolution of the coastline at different dates was done using DSAS version 4.3. The main results obtained show two evolutionary trends of coastal linear interest: in the long term, the study has given a decline in the coastline of an annual average rate equal to $-1.09 \mathrm{~m} /$ year between 1979 and 2018 and in the
\end{abstract}


medium term, the study shows an advance of the coastline with an average speed of evolution equal to $+0.75 \mathrm{~m} /$ year between 2000 and 2018 . In the same period, the zone experienced a short-term constant erosion between 2000-2005 and 2005-2008 with an average rate of $-1.12 \mathrm{~m} /$ year, a slight stability between 2008 and 2012 with a speed of $-0.08 \mathrm{~m} /$ year, while from 2012 to 2016 it has undergone an average evolution of $+2.44 \mathrm{~m} / \mathrm{year}$. A forecast of the phenomenon in 2028 has given a coastline position of $10.90 \mathrm{~m}$ compared to its current position, which could jeopardize hotel infrastructure such as: Seme Beach Hotel; Madison Beach; Etisah Beach and the fishing camps of Batoké village during episodes of high tide.

Keywords: Fattening, Erosion, Stability, Remote sensing, Rate of evolution

\section{Introduction}

L'érosion côtière est un phénomène général dans le monde (Paskoff, 1998), soit 70\% des plages de la planète touchée par ce phénomène (Bird, 1985). Ainsi en Europe, l'érosion touche $40 \%$ des plages de l'Union Européenne (European Commission, 2004) et plus de $50 \%$ des côtes sableuses en France métropolitaine (Ifen, 2006). D'après les travaux du GIEC (2007), les milieux côtiers sont attractifs et concentrent de très nombreux enjeux tels que des centres urbains, des activités économiques ou encore des complexes touristiques. Ces atouts font de la zone côtière, une zone fragile, car sujette à une forte pression humaine, leurs activités ont une grande influence sur le climat global avec l'émission de plus en plus importante des gaz à effet de serre dans l'atmosphère et donc l'une des conséquences serait la hausse actuelle et future du niveau de la mer. Certains auteurs (Ibe \& Awosika, 1991 ; Niang-Diop, 1993 ; Dennis et al., 1995) pensent qu'au vu de cette dynamique climatique déclinante, les problèmes d'érosion devraient s'aggraver surtout sur les côtes sableuses ouest- africaines déjà en recul. Par exemple, une élévation du niveau de la mer de $1 \mathrm{~mm} / \mathrm{an}$ entraînerait, en un siècle, selon le principe de Bruun, un recul de 264 à $839 \mathrm{~m}$ sur les plages gambiennes (Jallow et al., 1999). A l'instar des pays côtiers du monde, les littoraux de la zone du golfe de guinée sont également confrontés à ce phénomène, car dans les secteurs sableux, les taux d'érosion sont estimés par Ibe \& Quelennec (1989) entre 1,2 et $6 \mathrm{~m} / \mathrm{an}$. Les paysages côtiers du Golfe de Guinée sont confrontés aux grands problèmes environnementaux, tant d'origine naturelle qu'anthropique et l'érosion côtière en est une illustration (Yao et al., 2010). Blivi (2001), suggère que dans tous les pays du golfe de guinée, le problème d'érosion est pris sérieusement en compte par les chercheurs et les autorités administratives du fait de leurs conséquences très souvent désastreuses. Les auteurs (Ibe, 1988 ; Rossi, 1989 ; Blivi, 1993 ; Paskoff, 1993) pensent que les taux de recul de la ligne de rivage très importants, sous l'influence des facteurs 
hydrodynamiques, sont également à mettre en relation avec les grands ouvrages maritimes construits sur certaines portions du littoral, dans le cadre des programmes de développement économique des pays. Le littoral camerounais n'est pas épargné par les problèmes que subissent les pays côtiers du monde. Ainsi, pour un meilleur aménagement du littoral Camerounais, il convient de mieux connaitre le taux d'évolution du trait de côte, afin de prévoir et gérer les éventuels risques côtiers à venir. Les plages allant de Batoké à Seme Beach (côte ouest Camerounaise) sont sous l'emprise des agents hydrodynamiques (marée, houle, courant...) et des activités anthropiques (décapage du haut de plage, construction des ouvrages de protection), mais très peu de travaux ont été retrouvés dans la littérature traitant de la dynamique du trait de côte dans cette localité. Pour contribuer à fournir une banque de données sur le sujet, il est nécessaire d'étudier la dynamique du trait de côte sur la côte ouest du Cameroun (Batoké-Seme Beach) à l'aide des images satellitaires Landsat 3,7 et 8. Cette étude permettra de mieux connaitre le taux d'évolution du trait de côte, afin de prévoir et gérer les éventuels risques côtiers à venir. Ainsi, l'objectif général de l'étude est d'apporter une contribution à la connaissance de la dynamique du trait de côte de la bande côtière de la côte Ouest camerounaise allant de Batoké à Seme Beach en intégrant les mesures par télédétection. Plus spécifiquement, il s'agira de délimiter la portion d'intérêt sur l'image satellitaire de 2018, de déterminer la dynamique évolutive du trait de côte de la portion d'intérêt à moyen et long terme en utilisant les images fournies par les capteurs des Landsat 3, 7 et 8 de 1979 à 2018 et d'évaluer le taux global annuel à long terme de l'évolution du trait de côte de la portion d'intérêt de 1979 à 2018, puis simuler son évolution jusqu'en 2028.

\section{Materiel et Methode \\ Présentation du site d'étude}

Le site d'étude est situé au pied du Mont Cameroun entre les latitudes $4^{\circ} 2^{\prime}$ ' $10,7232^{\prime \prime}$ ' et $4^{\circ} 2^{\prime} 8,710436^{\prime \prime} \mathrm{N}$ et longitudes $9^{\circ} 5^{\prime} 41,94763$ ' 'et $9^{\circ} 5^{\prime} 41$, 8972" Est. Il est donc recouvert de coulées basaltiques issues de ses différentes éruptions. Les minéraux de ses roches sont hydrolysés par les eaux pluviales chaudes et abondantes, définissant un sol de type ferralitique (Muller \& Gavaud, 1979). Le climat régnant dans la zone d'étude est de type tropical côtier avec deux saisons : une longue saison de pluie allant de février à novembre avec juillet le mois le plus pluvieux $(720 \mathrm{~mm})$ et une courte saison sèche allant de décembre à janvier avec mars le mois le plus chaud $\left(26,4^{\circ} \mathrm{C}\right.$ température moyenne). (Source : www. climate-data.org).

La Figure 1 ci-dessous montre l'étendue de la bande côtière considérée dans le cadre de cette étude par superposition de la ligne de rivage instantanée 
obtenue sur le terrain avec le GPS, à une image satellitaire OLI/TIRS de Landsat 8 acquise en Mai 2018.

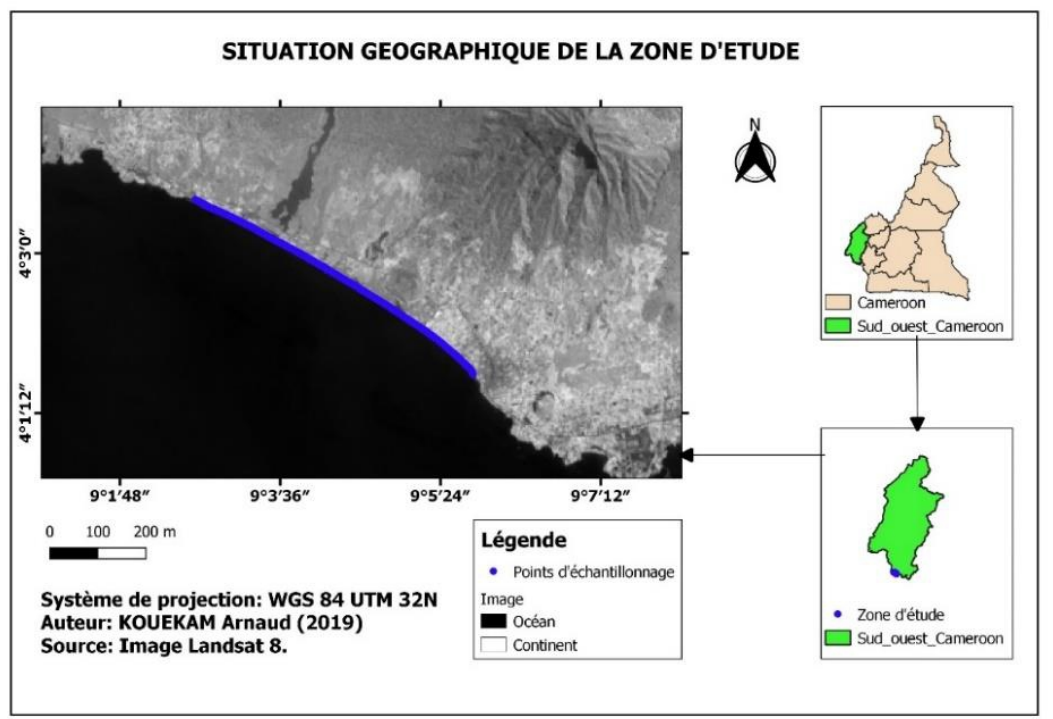

Figure 1. Localisation spatiale de la portion d'intérêt

\section{Matériels}

$\mathrm{Au}$ cours de cette étude, un GPS de marque Garmin de la série MONTANA 650T a été utilisé pour délimiter la portion d'intérêt, sept scènes Landsat datant de 1979 à 2018 sont utilisées pour l'analyse locale de la dynamique du trait de côte des plages sableuses (Tableau 1). Elles sont disponibles et téléchargeables gratuitement via les sites de l'USGS EarthExplorer (http//www.earthexplorer.usgs.org). Les outils appropriés du logiciel ENVI 5.1 sont utilisés pour le prétraitement des différentes scènes acquises, alors que l'extension Digital Shoreline Analysis System (DSAS 4.3) d'ArcGIS 10.1 est exploitée pour calculer les vitesses d'évolution du trait de côte.

\begin{tabular}{|c|c|c|c|}
\hline Satellite & Capteur & $\begin{array}{c}\text { Date } \\
\text { d'acquisition }\end{array}$ & $\begin{array}{c}\text { Résolution } \\
\text { spatiale }\end{array}$ \\
\hline Landsat 3 & MSS & $02 / 01 / 1979$ & $79 \times 79 \mathrm{~m}$ \\
\hline Landsat 7 & ETM+ & $10 / 12 / 2000$ & $30 \times 30 \mathrm{~m}$ \\
\hline Landsat 7 & ETM+ & $22 / 11 / 2005$ & $30 \times 30 \mathrm{~m}$ \\
\hline Landsat 7 & ETM+ & $31 / 01 / 2008$ & $30 \times 30 \mathrm{~m}$ \\
\hline Landsat 7 & ETM+ & $26 / 01 / 2012$ & $30 \times 30 \mathrm{~m}$ \\
\hline Landsat 8 & OLI/TIRS & $21 / 01 / 2016$ & $30 \times 30 \mathrm{~m}$ \\
\hline Landsat 8 & OLI/TIRS & $26 / 01 / 2018$ & $30 \times 30 \mathrm{~m}$ \\
\hline
\end{tabular}

Tableau 1. Caractéristiques des scènes Landsat utilisées 


\section{Méthode}

\section{Levés au GPS}

Les levés au GPS ont eu pour but dans un premier temps de prendre les coordonnées géographiques in situ de la position du trait de côte matérialisant la zone d'intérêt et de la localiser sur les différentes images satellitaires faisant l'objet de l'étude et dans un second temps de prendre les coordonnées géographiques des différents sites d'intérêt économique situés sur la portion d'intérêt afin d'apprécier leurs vulnérabilités. Ainsi la bande côtière considérée a été parcourue à l'aide d'un GPS de marque Garmin de la série MONTANA 650T, qui a été programmé de façon qu'à chaque seconde celui-ci enregistre les coordonnées géographiques de la position du trait de côte, paramétré sur mode suivi de trajet et introduit dans un sac à dos. Après cette manœuvre, le porteur de GPS a parcouru la laisse instantanée la plus humectée matérialisant ici le trait de côte, sans arrêt du point de départ jusqu'au point d'arrivé où celui-ci ressortait le GPS pour arrêter l'enregistrement. Les coordonnées obtenues ont été superposé sur l'image satellitaire de 2018 juste pour s'assurer qu'ils correspondent à la limite terre/mer (fig. 1).

\section{Prétraitement des images}

Le prétraitement des images a consisté en la correction atmosphérique afin de minimiser les effets d'altération au niveau des valeurs radiométriques généré par l'interpolation pendant la correction géométrique. En premier lieu, nous avons procédé à une calibration radiométrique suivant la radiance de chaque image ; cela nous a permis de comparer les images multi temporelles et après la calibration radiométrique, à l'aide de l'outil FLAASH (Fast Lineof-sight Atmospheric Analysis Of Hypercube), nous avons procédé à une correction atmosphérique dont le but est d'atténuer l'effet atmosphérique, de compenser les différences des conditions de prise de vues et de calibrer les capteurs.

\section{Mesure de la vitesse d'évolution du trait de côte}

L'extraction de la ligne de rivage instantané (trait de côte) a été préalablement effectuée. Les ratios de bandes développés par Guariglia et al (2006) et Restrepo (2012) ont été utilisés dans ce travail de recherche car donnant la meilleure discrimination de l'interface terre/mer. Le principe est le suivant: nous avons exploité les bandes du moyen infrarouges (la bande 4 pour les images MSS de Landsat 3, la bande 5 de ETM+ pour Landsat 7 et la bande 6 de OLI/TIRS pour les Landsat 8) et les bandes correspondant au vert (bande 1 de MSS pour Landsat 3, bande 2 de ETM+ pour Landsat 7 et bande 3 de OLI/TIRS pour Landsat 8). Ensuite, pour extraire la ligne de rivage instantanée (trait de côte) à partir des images Landsat couvrant la période entre 
1979 et 2018, nous avons procédé au ratio B5/B2 de ETM+/Landsat 7 (Guariglia et al., 2006) et B6/B3 de OLI/TIRS/ Landsat 8. Cependant, dans la situation de l'unique image Landsat MSS, le ratio B4/(B2-B1) utilisé par Guariglia et al (2006) est retenu. La vitesse d'évolution du trait de côte est évaluée entre deux dates et la méthode statistique End Point Rate (EPR) est retenue pour calculer la vitesse d'évolution du trait côte dans le logiciel « Digital Shortline Analysis System (DSAS) ». Après avoir obtenu la vitesse d'évolution du trait de côte, la simulation de l'évolution du trait de côte a été effectué automatiquement avec le logiciel ArcGis 10.1 en se servant de l'outil Buffer.

\section{Resultats et Discussion \\ Resultats}

Variation spatio-temporelle du trait de côte de 1979 à 2018

L'analyse de la variation à moyen terme de l'évolution du trait de côte de 2000 et 2018 de la portion d'intérêt montre 04 secteurs caractérisés par un phénomène d'érosion avec des vitesses sectorielles comprises entre $-2,52 \mathrm{~m} / \mathrm{an}$ et $-7,64 \mathrm{~m} / \mathrm{an}$. La zone d'intérêt a également connu à cette même période 03 secteurs en engraissement avec des vitesses sectorielles comprises entre $+1,46$ $\mathrm{m} / \mathrm{an}$ et $+24,29 \mathrm{~m} / \mathrm{an}$ (fig.2). à long terme, la variation de l'évolution du trait de côte de 1979 et 2018 de la portion d'intérêt montre que tous les secteurs de la zone d'étude sont en érosion avec des vitesses sectorielles comprises entre $-1,73 \mathrm{~m} /$ an et $-3,83 \mathrm{~m} /$ an (fig. 3 ). 


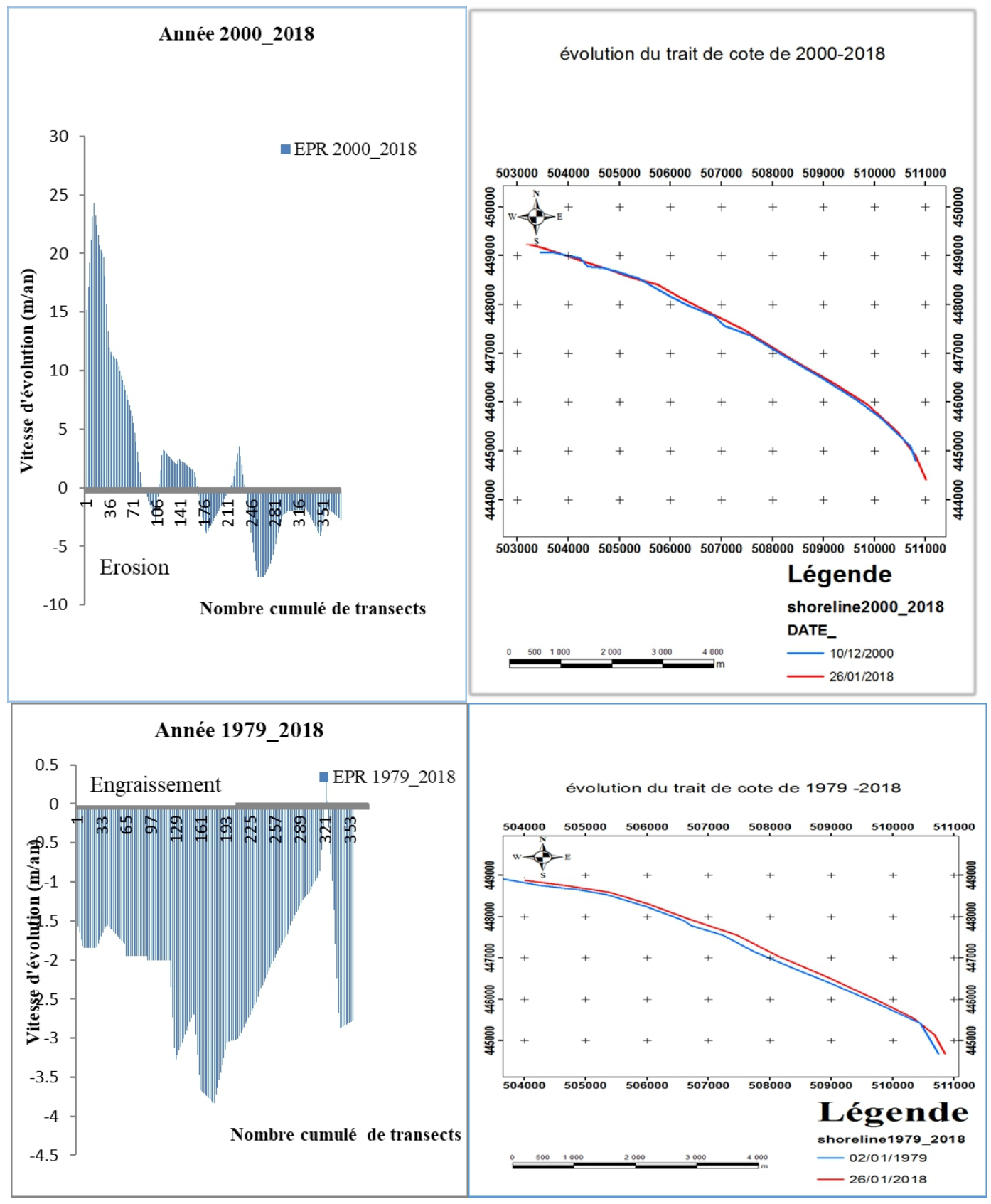

Figure 3. Évolution des vitesses d'érosion et d'engraissement des secteurs du trait de côte entre 1979 et 2018 par la méthode des points extrêmes (EPR) 


\section{Bilan évolutif du trait de côte le long du linéaire côtier considéré entre 1979 et 2018}

Le tableau 2 ci-dessous montre l'évolution diachronique globale du trait de côte de 1979 à 2018. Il en ressort que la bande côtière d'intérêt est identifiée en moyenne comme une zone en érosion du moins pour la période considérée, avec une vitesse moyenne d'érosion égale à -1,09 m/an. Cette zone est marquée par une érosion entre 2000-2005 et 2005-2008, avec des taux moyens constant de $-1,12 \mathrm{~m} / \mathrm{an}$, une légère stabilité entre 2008-2012 avec un taux moyen de $-0,08 \mathrm{~m} / \mathrm{an}$, tandis que de 2012 à 2016, elle a subi un engraissement en moyenne de $+2,44 \mathrm{~m} / \mathrm{an}$ et entre 2000 et 2018 elle a subi un engraissement en moyenne de $+0,75 \mathrm{~m} / \mathrm{an}$.

\begin{tabular}{|c|c|c|}
\hline Années & Phénomènes & Evolution de la côte (m/an) \\
\hline $2000-2005$ & Erosion & $-1,12$ \\
\hline $2005-2008$ & Erosion & $-1,12$ \\
\hline $2008-2012$ & Stabilité & $-0,08$ \\
\hline $2012-2016$ & Engraissement & $+2,44$ \\
\hline $2000-2018$ & Engraissement & $+0,75$ \\
\hline $1979-2018$ & Erosion & $-1,09$ \\
\hline
\end{tabular}

Tableau 2. Synthèse de l'évolution du trait de côte du secteur d'étude.

\section{Prévision de la position du trait de côte de 2018 à 2028}

La prévision de la position du trait de côte est évaluée à partir de la formule de la vitesse V=Distance/Temps écoulé entre 2018 et 2028. En considérant comme origine l'année 2018 avec une vitesse d'évolution de recul du trait de côte égale à $-1,09$, il en ressort qu'en supposant cette vitesse moyenne d'évolution constante à cette date, le trait côte reculera à une distance de 10,90 m en 2028 par rapport à sa position actuelle. La figure 4 montre un essai de modélisation de la position du trait de côte à partir d'un Buffer de rayon égal à $10,90 \mathrm{~m}$. Le trait de cote étant en moyenne en recul (vitesse négative), la partie supérieure du Buffer dirigée vers le continent représente alors le trait de côte en 2028, et les points sur la figure représentent les sites économiquement vulnérables à cette date qui sont presque en contact avec le tait de côte de 2028. Ainsi les infrastructures telles que ; Semé Beach Hôtel ; Madison Hôtel ; Etisah Beach et les campements des pêcheurs situés sur la portion du trait de côte d'intérêt pourraient être alors envahi par les eaux pendant les épisodes de marées de vives eaux. 


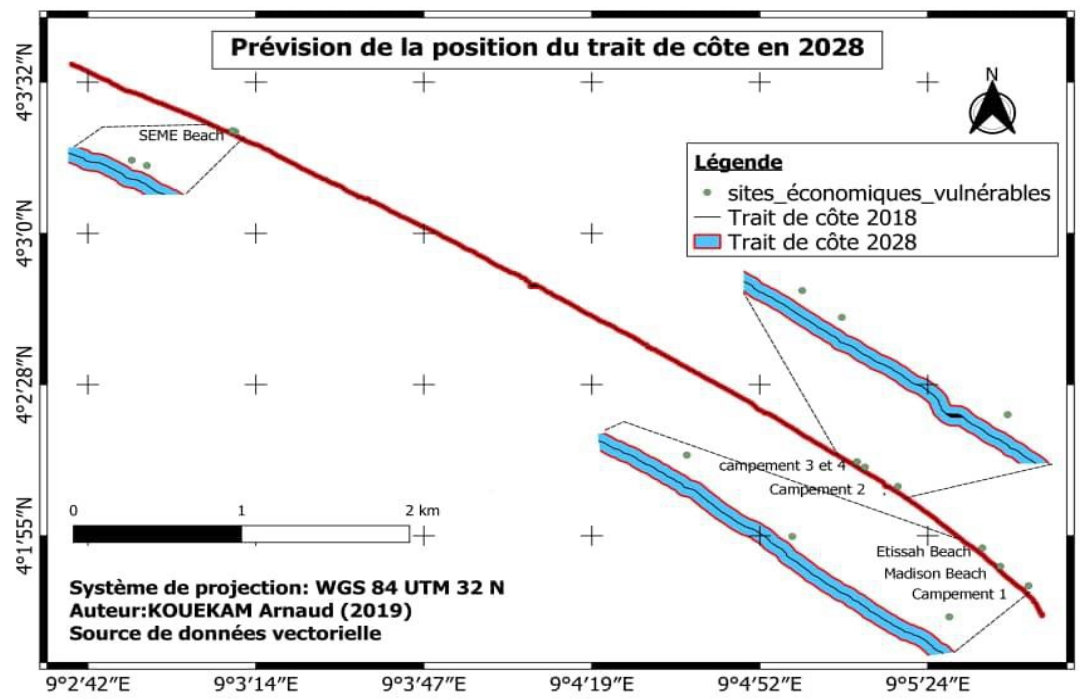

Figure 4. Modélisation de la position du trait de côte et infrastructures menacées en 2028

\section{Discussion}

Les analyses des images satellitaires par extraction et superposition des différentes couches vectorielles ont permis de qualifier l'évolution à moyen terme suite à la détermination des taux globaux annuels du trait de côte de 2000 à 2018. Les résultats obtenus durant cette période montrent que la bande côtière considérée est sur l'emprise d'un engraissement d'une valeur moyenne de $+0,75 \mathrm{~m} / \mathrm{an}$, ce résultat est supérieur à celui obtenu par Mouctchou et al. (2014) au nord de l'embouchure de l'oued Tighissasse au Maroc. Cette différence peut s'expliquer par le fait que les auteurs ont utilisés des feuilles topographiques pour l'étude de l'évolution historique du trait de côte.

Une étude sur 4 décennies a été effectuée en vue d'appréhender l'évolution du trait de côte sur le long terme (1979-2018) et le résultat témoigne qu'en général la portion d'intérêt est de manière moyennée en recul avec une valeur de $-1,09 \mathrm{~m} / \mathrm{an}$. Ce résultat est inférieur à la moyenne de déplacement de $-1,13 \mathrm{~m} / \mathrm{an}$ retrouvée par Djagoua et al (2016) à proximité du port de Cap de l'Eau. Cette différence serait certainement due à la forte anthropisation du secteur. Le résultat obtenu lors de la simulation en considérant le taux global moyen de recul à la date $\mathrm{t}_{0}$ (Année 2018) constant vaudra $10,90 \mathrm{~m}$ en 2028 , il est inférieur à la valeur de $13,53 \mathrm{~m}$ prédit par Akadje (2010) sur le port de Bouet en Côte d'Ivoire, cela peut s'expliquer par la différence de pas de temps alloué à la prédiction et la vitesse considérée pour la simulation. 


\section{Conclusions et Perspectives}

L'étude de la dynamique évolutive du trait de côte sur la portion de la côte ouest du Cameroun allant de Batoké à Seme Beach (Limbé) a été initiée dans le but de caractériser l'évolution historique de la ligne de rivage afin, de prévoir la gestion durable des zones côtières ouest camerounaises. Les résultats obtenus montrent que la bande côtière considérée est gouvernée par 2 tendances évolutives du trait de côte : d'un recul moyen de $-1,09 \mathrm{~m} / \mathrm{an}$ avec un léger engraissement entre 2000 et 2018 d'une valeur de $+0,75 \mathrm{~m} / \mathrm{an}$. La simulation du phénomène en 2028 a permis d'avoir un recul de la position du trait de côte de 10,90 m par rapport à sa position actuelle, ce qui pourrait mettre en péril les infrastructures hôtelières telles que : Seme Beach Hôtel ; Madison Beach ; Etisah Beach et les campements des pêcheurs du village Batoké lors des épisodes de marées de vives eaux. Pour éviter que cela ne devienne réalité à l'avenir, il serait opportun pour les chercheurs de l'IRAD de Batoké de proposer à la communauté urbaine de Limbé un plan d'occupation des sols dans la localité de Limbé et des mesures d'atténuation souples pour les populations déjà installées. ce travail permettra de disposer d'une base de données pour le suivi de l'évolution du trait de côte sur la côte ouest du Cameroun (Limbé), mais mérite d'être complété par d'autres investigations notamment dans l'avenir sur des travaux complémentaires tels que : le Suivi par télédétection de la variabilité du trait de côte, la cartographie des zones en déséquilibre et l'analyse des causes de la dynamique sur toute la côte OuestCameroun et également une étude évolutive de l'occupation des sols en lien avec la position passée et actuelle du trait de côte à l'aide des images satellitaires sur la côte Ouest camerounaise doit être envisagée.

\section{References:}

1. Akadjé, L. (2010). Analyse de la cinématique du trait de côte de portBouet à l'aide du couplage télédétection et SIG. Mémoire de DEA, Université Cocody, Abidjan, Côte d'Ivoire, 42p.

2. Bird, C. (1985). Coastline changes a global review. John Wiley \& Sons Chichester, New York, Brisbane, Toronto, Singapore, 219 p.

3. Blivi A. (1993). Erosion côtière dans le golfe de Guinée en Afrique de l'Ouest : exemple du Togo. Université de Lomé, Togo, 9p.

4. Blivi, A. (2001). Impact de l'érosion côtière et éléments d'étude de la vulnérabilité : Exemple du Togo (Golfe de Guinée). Revue de 1'Université de Moncton. $n^{\circ} 1-2$, pp 289-308.

5. Dennis, K.C., Niang-Diop I., Nicholls R.J. (1995). Sea-level rise and Senegal: Potential impacts and consequences. Journal of Coastal Research, Special Issue n²14, pp. 243-261.

6. Djagoua, E.M.V., Bakayoko, F., Kouadio, M.J., Kassi A.J.B., Mobio, A.B.H., Adonis K. K., Tiemélé J.A., Yavo, P. (2016). Cartographie de 
la dynamique Du Trait De Côte A Grand-Lahou: Utilisation De L'outil « Digital Shoreline Analysis System (Dsas)». European Scientific Journal, edition vol.12, No.36, PP. 327 - 335.

7. European Commission (2004). Living with coastal erosion in Europe: sediment and space for sustainability. Part II Maps and statistics. Report Directorate General of Environment, European Commission, Brussels, 25 p.

8. Guariglia, A., Buonamassa, A., Losurdo, A., Saladino, R., Trivigno, M.L., Zaccagnino, A., Colangelo, A. (2006). A multisource approach for coastline mapping and identification of shoreline changes. Annals of Geophysics, $\mathrm{n}^{\circ}$ 49, pp. 295-304.

9. Ibe, A.C., (1988). Nigeria In Walker, Artificial structures and shorelines. H.J. (ed) Kluwer Acad. Pub., Dordrecht, pp. 287-294.

10. Ibe, A.C., Awosika, L.F. (1991). Sea level rise impact on african coastal zone. In (Omide S.H., Juma C., Eds). A change in the weather: African perspectives on climate change. Nairobi, Kenya, African center for technology studies, Nairobi, pp. 105-112.

11. Ibe, A.C., Quelennec, R. E., (1989). Méthodologie d'inventaire et de contrôle de l'érosion côtière dans la région de l'Afrique de l'Ouest et du Centre. Rapport et études des mers regionals, $\mathrm{n}^{\circ} 107.107 \mathrm{p}$.

12. Ifen (2006). Un quart du littoral recul du fait de l'érosion. Les 4 pages de l'IFEN, $\mathrm{n}^{\circ} 13,4 \mathrm{p}$.

13. Jallow, B.P., Toure, S., Barrow, M., Mathieu, A.A. (1999). Coastal zone of the Gambia and the Abidjan region in Côte d'Ivoire: sea level rise vulnerability, response strategies, and adaptation options. Climate Research, $\mathrm{n}^{\circ} 12$, pp. 129 - 136.

14. Moutchou, B., Benali, B., Mamouni, A., (2014). Evolution diachronique de la ligne du rivage le long des littoraux méditerranéens occidentaux marocains : cas des sites côtiers de Ksar Esghir, Bou Ahmed et Jebha (Maroc). Edition paralia CFL vol n8, pp. 317-324.

15. Muller, J.P., Gavaud, M. (1979). Atlas de la République unie du Cameroun, édition jeune Afrique. Paris, pp. 25-26.

16. Niang-Diop, I. (1995). L'érosion côtière sur la petite côte du Sénégal à partir de l'exemple de Rufisque. Passé, présent, futur. Thèse, Université d'Angers, UFR Environnement. n 110. pp 675-383.

17. Paskoff R. (1998). La crise des plages : pénurie de sédiments. Mappemonde, $\mathrm{n}^{\circ} 4$, pp. 11-15.

18. Paskoff, R. (1993). Côtes en danger. Masson, Paris, 250 p.

19. Rossi G., (1989). L'érosion du littoral dans le Golfe du Bénin : un exemple de perturbation de l'équilibre dynamique. Zeitschrift für Geomorphologie, Supplement Band, n73, pp. 139-165. 
20. Restrepo, J.D. (2012) - Assessing the effect of sea-level change and human activities on a major delta on the Pacific coast of northern South America: The Patía River. Geomorphology, n ${ }^{\circ} 10$ pp. 612-619

21. Yao, K.S., Abe J., Bamba, S.B., Konan, K.E., Aka, K., (2010). Dynamique d'un périmètre littoral portuaire : la côte de San-Pédro, Sud-Ouest de la Côte d'Ivoire. Revue Paralia, n ${ }^{\circ}$, pp 227251. DOI :10.5150/revue-paralia.2010. 